\title{
Restoration of Homeostasis in the Tracheal Mucosa After Thyroid Surgery in a Rat Model
}

\author{
BO HAE KIM ${ }^{1,2}$, HEE-BOK KIM ${ }^{1}$, JOO HYUN PARK ${ }^{1,2}$, CHANG GUN CHO ${ }^{1,2}$, \\ SEOK-WON PARK ${ }^{1,2}$ and YUN-SUNG LIM ${ }^{1,2}$ \\ ${ }^{1}$ Department of Otorhinolaryngology-Head and Neck Surgery, \\ Dongguk University Ilsan Hospital, College of Medicine, Goyang, Republic of Korea; \\ ${ }^{2}$ Sensory Organ Research Institute, College of Medicine, Dongguk University, Gyengju, Republic of Korea
}

\begin{abstract}
Background/Aim: This study aimed to investigate the process of homeostatic restoration in the tracheal mucosa (TM) after thyroid surgery. Materials and Methods: Fifty-four rats were divided into normal controls $(N C)$ and three experimental groups: (i) flap elevation (FE), (ii) thyroid exposure (TE), and (iii) thyroid isthmusectomy (TI). Expression of $m R N A$ and proteins of key factors regulating homeostasis were evaluated in the TM obtained 3, 7, and 21 days after thyroid surgery. Results: Increased mRNA expression of transforming growth factor- $\beta 1$ (TGF- $\beta 1$ ), hypoxia-inducible factor-1 $\alpha(H I F-1 \alpha)$, and matrix metalloproteinase-9 (MMP-9) were observed 21 days after thyroid surgery in all experimental groups compared to that of NC group. Conclusion: Thyroid surgery leads to an actual increase of TGF- $\beta 1, H I F-1 \alpha$, and MMP-9 expression in the TM. This increased expression of key regulators of homeostatic restoration in the TM lasts for a considerable period of time after surgery, especially if the extent of surgery increased.
\end{abstract}

The respiratory mucosa is composed of pseudostratified columnar epithelium, lamina propria, submucosa containing rich mucus-secreting glands, and well-developed capillary plexus (1). Although the respiratory mucosa is frequently

This article is freely accessible online.

Correspondence to: Yun-Sung Lim, Department of Otorhinolaryngology-Head and Neck Surgery, Dongguk University Ilsan Hospital, College of Medicine, 27 Dongguk-ro, Ilsandong-gu, Goyang, 10326, Republic of Korea. Tel: +82 319617439, Fax: +82 319617437, e-mail: yslim0503@gmail.com and Seok-Won Park, Department of Otorhinolaryngology-Head and Neck Surgery, Dongguk University Ilsan Hospital, College of Medicine, 27 Dongguk-ro, Ilsandong-gu, Goyang, 10326, Republic of Korea. Tel: +82 319617430, Fax: +82 319617427, e-mail: sw43857@dumc.or.kr

Key Words: Thyroidectomy, respiratory mucosa, trachea, transforming growth factor beta, hypoxia. exposed to mechanical or chemical stimuli because of the nature of the airway, it maintains adequate homeostasis via harmonious and dynamical response to inflammatory stimuli in the overall structure of the respiratory mucosa $(2,3)$.

The homeostatic restoration of respiratory mucosa is initiated by inflammatory cell infiltration into the injured respiratory mucosa, and is regulated by a complex molecular pathway (3). The inflammation induced in the injured tissue is modulated by various cytokines, growth factors, and other molecules leading to changes in epithelial cells, surrounding stroma, and submucosa (3). Blood and lymphatic vessels play a critical role in the entire process of restoring tissue homeostasis. However, chronic stimulation triggers inflammation in the respiratory mucosa and eventually leads to structural and functional changes across all components of the respiratory mucosa, which is observed as a loss of epithelial barrier, basement membrane thickening, subepithelial fibrosis, glandular hyperplasia, increased vascularity, and edema $(2,4)$.

We previously reported mucosal thickening with submucosal glandular hyperplasia in tracheal mucosa (TM) combined with fibrin deposition following thyroid surgery, resulting in increased mucus production and basal cell activation (5). Since the blood and lymphatic fluid of the TM drain into the deep neck space via the connected vessels is located around the thyroid glands $(6,7)$, we suggested that edema induced by thyroid surgery might be one of the mechanisms underlying changes in TM, resulting in delayed homeostatic restoration of the TM (5). Although TM changes following thyroid surgery are expected to eventually restore homeostasis, these changes are similar to those observed in remodeling of small airway. Incomplete recovery of TM can cause prolonged discomfort of throat known as postthyroidectomy syndrome (5).

The aims of this study were to investigate the process of homeostatic restoration in the tracheal mucosa (TM) after thyroid surgery by evaluating mRNA and protein expression of key regulators of homeostasis and determine the impact of the extent of thyroid surgery on the homeostatic restoration of TM. 


\section{Materials and Methods}

Study design and key regulators of the restoration of homeostasis. This animal study was approved by the Institutional Animal Care and Use Committee of our Institution (IACUC No. 2017-08166). Fiftyfour female Sprague-Dawley rats weighing 200 to $250 \mathrm{~g}$ each (Orient Bio, Republic of Korea) were purchased and housed for a week in a pathogen-free animal facility under controlled 12-h light-dark cycle, at $21-23^{\circ} \mathrm{C}$ and $40-60 \%$ relative humidity with free access to food pellets and water. The rats were randomly divided into four groups, with nine rats in the normal control (NC) group and 15 rats in each experimental group. The experimental groups were classified into three groups based on stage and extent of thyroid surgery as follows: (i) skin incision followed by flap elevation (FE); (ii) thyroid exposure via midline division between strap muscles (TE); and (iii) thyroid isthmusectomy (TI). The reason for performing only thyroid isthmusectomy, but not total or hemi-thyroidectomy, was to minimize the risk of the impaired wound healing caused by hypothyroidism.

Since morphological and functional changes of TM tended to recover in four weeks after thyroid surgery in our previous study (5), three rats in the NC groups and five rats in each experimental group were euthanized to harvest TM at three (D3), seven (D7), and 21 (D21) days after thyroid surgery. We defined D3 as the early phase, D7 as the intermediate phase, and D21 as the late phase postthyroid surgery.

Transforming growth factor- $\beta 1$ (TGF- $\beta 1$ ) which is known to affect multiple structural cells during inflammation and the homeostatic restoration was used as a major marker of TM changes following thyroid surgery (8). TM hypoxia caused by tissue damage and inflammation was evaluated using hypoxia-inducible factor- $1 \alpha$ (HIF-1 $\alpha$ ) which dynamically responded to oxygen deficiency in the tissue (9). Matrix metalloproteinase-9 (MMP-9) is a transcriptional factor expressed during airway remodeling in the epithelial basement membrane (10). Microcirculatory changes in the blood and lymphatics were evaluated using markers of platelet endothelial cell adhesion molecule-1 (CD 31 also known as PECAM-1) for angiogenesis and vascular endothelial growth factor receptor-3 (VEGFR-3) for lymphangiogenesis.

Surgical procedures and histological evaluation. The surgical procedure was generally carried out as described in a previous study (5). All rats in both $\mathrm{NC}$ and experimental groups were anesthetized using an intramuscular injection containig $0.1 \mathrm{mg} / \mathrm{kg}$ of tiletamine hydrochloride/zolazepam hydrochloride (Zoletil 50, Vibac Laboratories, Carros, France) and $0.1 \mathrm{mg} / \mathrm{kg}$ xylazine (Rompun, Bayer, Leverkusen, Germany). The NC group was awakened without a surgical procedure. Briefly, all surgical procedures were performed under aseptic conditions based on the procedures reported in a previous study. After preparation of the anterior neck using povidone-iodine solution, a vertical incision was made at the midline of the neck, followed by exposure of the full length of the strap muscles by flap elevation (FE). Thereafter, the thyroid gland and cervical trachea were exposed following the midline division between both strap muscles (TE). We eventually resected the thyroid isthmus separated by $2 \mathrm{~mm}$ width (TI). Bleeding was controlled by light compression to minimize compressive or thermal injury to the trachea.

An aero-digestive tract between the level of the larynx and the cervical trachea including the strap muscles was obtained at each time point postoperatively, and the soft tissue including the strap
Table I. Primer sequences for quantitative real-time PCR.

\begin{tabular}{llc}
\hline Gene & Orientation & Sequence $\left(5^{\prime}-3^{\prime}\right)$ \\
\hline GAPDH & Forward & AGTTCAACGGCACAGTCAAG \\
& Reverse & TACTCAGCACCAGCATCACC \\
TGF- $\beta 1$ & Forward & CACTCCCGTGGCTTCTAGTG \\
& Reverse & CTGGCGAGCCTTAGTTTGGA \\
HIF1- $\alpha$ & Forward & AATCTGAGGACACGAGCTGC \\
& Reverse & GCTGCCGAAGTCCAGTGATA \\
VEGFR3 & Forward & CAGACCCACACGGAACTCTC \\
& Reverse & GTCCTTTGAGCCACTCGACA \\
CD31 & Forward & CCCAGTGACATTCACAGACA \\
& Reverse & ACCTTGACCCTCAGGATCTC \\
MMP9 & Forward & TCGGATGGTTATCGCTGGTG \\
& Reverse & AAGACGCACATCTCTCCTGC
\end{tabular}

GAPDH: Glyceraldehyde 3-phosphate dehydrogenase; TGF- $\beta 1$ : transforming growth factor- $\beta 1$; HIF- $1 \alpha$ : hypoxia-inducible factor- $1 \alpha$; CD 31: platelet endothelial cell adhesion molecule-1 (PECAM-1); VEGFR-3: vascular endothelial growth factor receptor-3; MMP-9: matrix metalloproteinase-9.

muscles attached to the cervical trachea was manually detached. After fixing with buffered $4 \%$ formalin for $24 \mathrm{~h}$, the trachea was embedded in paraffin and sectioned into $4 \mu \mathrm{m}$-thick slices, and stained with hematoxylin (Sigma-Aldrich, HHS32) and eosin Y (Sigma-Aldrich, E6003) (H\&E) to determine the structural changes, as reported in a previous study.

Quantitative real-time PCR. The quantitative measurement of mRNA expression of regulators, mentioned above, were evaluated in the harvested mucosa. The mRNA expression was analyzed via quantitative real-time polymerase chain reaction (qRT-PCR) in the TM harvested at the level of the thyroid isthmus. Total RNA was extracted from the TM of the experimental and control rats using TRIzol $^{\mathrm{TM}}$ Reagent (Invitrogen, Thermo Fisher Scientific, Inc., Waltham, MA, USA) according to the manufacturer's instructions. The RNA was transcribed to cDNA via cyclic temperature reverse transcription (AccuPower ${ }^{\circledR}$ CycleScript ${ }^{\mathrm{TM}}$ RT PreMix, Bioneer, Daejeon, Republic of Korea) with specific primers (Table I). GAPDH was used to normalize gene expression. qRT-PCR analysis was repeated three times for each specimen.

Immunofluorescence and immunohistochemical staining. Primary antibodies targeting TGF- $\beta 1$ (MA5-16949, Thermo Fisher, IL, USA), HIF-1 $\alpha$ (ab216842, Abcam, Cambridge, UK), and MMP-9 (ab228402, Abcam) were used and then coupled with secondary antibody (Alexa Fluor ${ }^{\circledR} 488$ goat anti-mouse IgG, A11001, Life Technologies, Carlsbad, CA, USA). Each image was captured by an Olympus BX53 microscope (Olympus, Tokyo, Japan) fixed with a DP73 cooled digital color camera (model DP73-1-51; Olympus). The protein expression was localized via immunofluorescence (IF) staining of TGF- $\beta 1$, HIF- $1 \alpha$, and MMP-9, and the mean fluorescence intensity (MFI) of each marker in TM was calculated and compared.

Immunohistochemical (IHC) staining of CD31 and VEGFR-3 was used to determine the newly formed vasculature and lymphatic vessels. The deparaffinized slide was treated with primary 


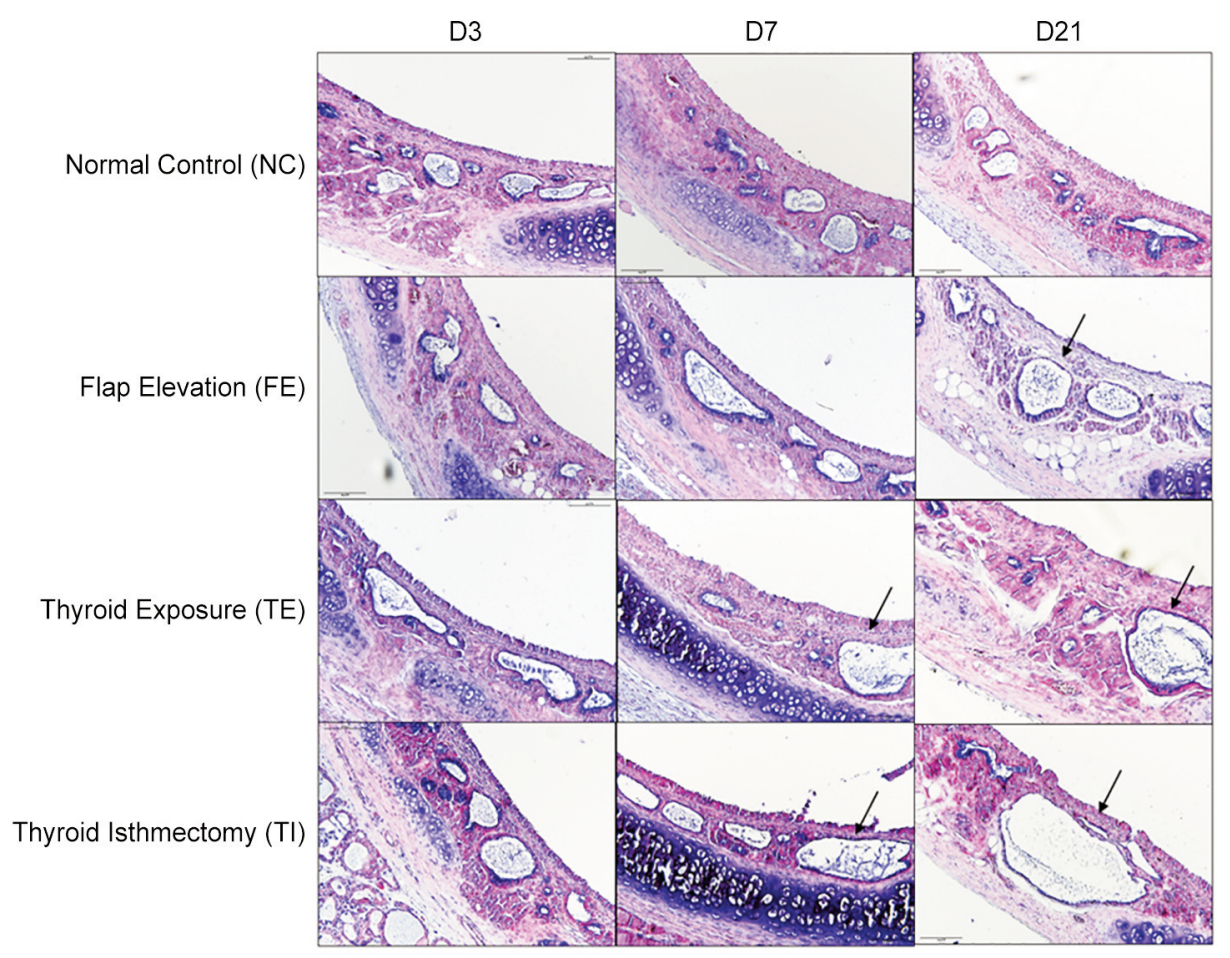

Figure 1. Representative images of the tracheal mucosa at each time point according to the surgical groups (H\&E stain '200 magnification). Submucosal gland hyperplasia is marked by black arrows.

antibodies against CD31 (ab64543, Abcam $\left.{ }^{\circledR}\right)$ and VEGFR-3 (ab27278, Abcam) and then incubated at $4^{\circ} \mathrm{C}$ for 24 h. 3, 3'Diaminobenzidine (DAB; Code K3468, Dako North America Inc., Carpinteria, CA, USA) staining was used to provide contrast

Statistical analysis. The normality of data evaluated by the ShaprioWilk test and the Mann-Whitney $U$-test was used to compare differences in mRNA expression and the MIF intensity. All statistical analyses were performed using IBM SPSS Statistics for Windows version 22.0 (IBM, Armonk, NY, USA). The null hypothesis of no difference was rejected if the $p$-Value was $<0.05$.

\section{Results}

All rats in both the NC and experimental groups survived without unexpected events for 21 days postoperatively, and $\mathrm{H} \& \mathrm{E}$ staining for TM revealed relative submucosal gland hyperplasia with a thickening of the TM in the experimental groups compared to the NC group at D21 (Figure 1). The time course of mRNA expression of key regulators after thyroid surgery is summarized in Figure 2.

TGF- $\beta 1$ expression. The TGF- $\beta 1$ mRNA expression in the TI group increased initially at D3 $(p=0.002)$ compared to that of the NC group, and the increased expression continued until D21 (D7, $p<0.001 ; \mathrm{D} 21, p<0.001)$. The relative increase in
TGF- $\beta 1$ mRNA expression compared to that in NC group was observed in the TE group at both D7 $(p<0.001)$ and D21 $(p<0.001)$ and in the FE group at only D21 $(p=0.006)$. A significant difference in the mRNA expression in the experimental groups was found between the FE and TI groups $(p<0.001)$ on D7, and the TGF- $\beta 1$ mRNA in the TI group was significantly higher than that in the FE $(p<0.001)$ and TE ( $p=0.019$ ) groups at D21 (Figure $3 \mathrm{~A}$ ). The expression of TGF- $\beta 1$ protein was dominant in the basal cell layer and submucosa 21 days after surgery (Figure 3B), the MFI value for TGF- $\beta 1$ of TI group at this time point was significantly higher than in other groups (NC group, $p<0.001$; FE group, $p=0.0049$; TE group, $p<0.001$ ) (Figure 3C).

HIF-1 $\alpha$ expression. HIF-1 $\alpha$ mRNA expression was significantly increased in the TI group $(p=0.031)$ compared to the NC from D3. All experimental groups showed increased HIF-1 $\alpha$ expression compared to the NC group at D7 (FE group, $p=0.019$; TE group, $p=0.046$; TI group, $p=0.006$ ), and the increased expression continued until D21 (FE group, $p<0.001$; TE group, $p<0.001$; TI group, $p<0.001$ ) without significant differences between the experimental groups (Figure 4A). The IF staining for HIF- $1 \alpha$ revealed that was HIF-1 $\alpha$ was expressed in all the components of TM, including epithelium, basal cell layer, and submucosa seven 

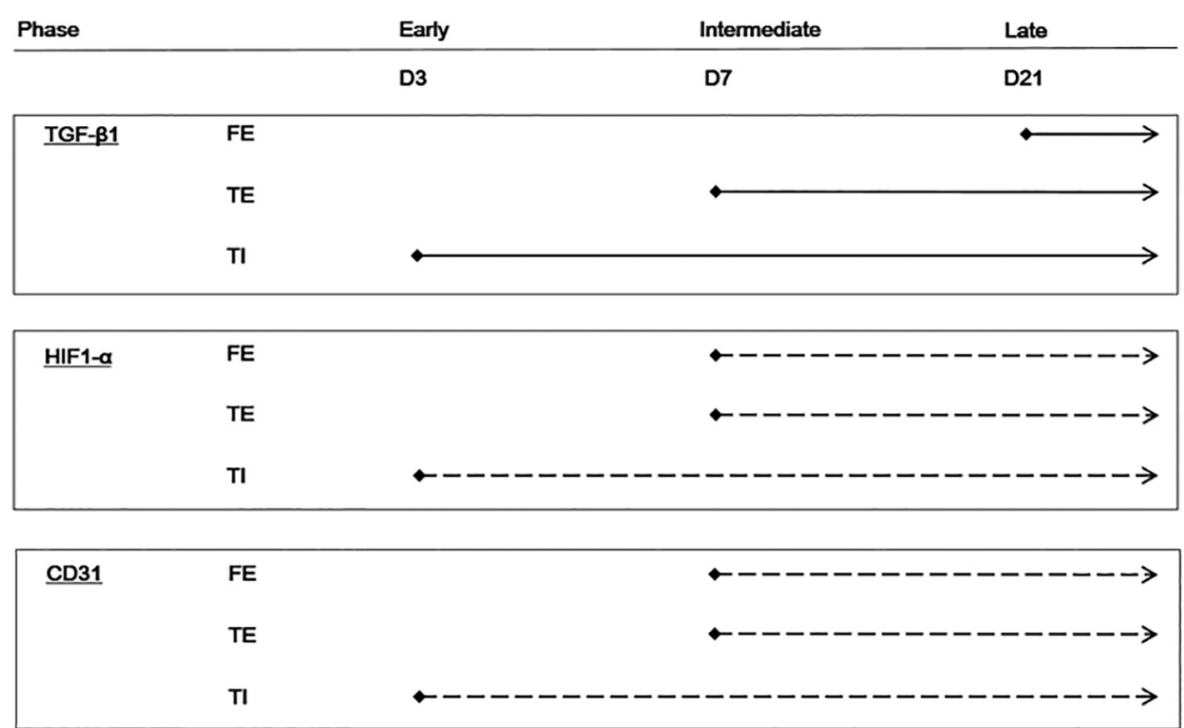

\begin{tabular}{|c|c|c|}
\hline \multirow[t]{3}{*}{ VEGFR-3 } & FE & $\bullet----\rightarrow$ \\
\hline & $\mathrm{TE}$ & $\bullet-------\rightarrow$ \\
\hline & $\mathrm{TI}$ & 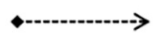 \\
\hline
\end{tabular}

\begin{tabular}{|c|c|c|}
\hline \multirow[t]{3}{*}{ MMP-9 } & $\mathrm{FE}$ & 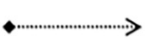 \\
\hline & $\mathrm{TE}$ & 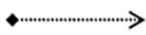 \\
\hline & TI & $\bullet$ \\
\hline
\end{tabular}

Figure 2. A brief presentation of the pattern of increased mRNA expression over time after thyroid surgery.

days after surgery (Figure 4B). The MFI value of the experimental groups showed a significant difference from that of the NC group (FE group, $p<0.001$; TE group, $p<0.001$; TI group, $p<0.001$ ), and the MFI of TE group was higher than in FE group $(p=0.001)$ and TI group $(p=0.001)$ (Figure 4C).

CD31 and VEGFR-3 expressions. CD31 mRNA expression was significantly increased in the TI group from D3 after surgery compared to that of the NC group ( $p=0.038)$, and the significantly increased expression continued until D21 in the TI group (D7, $p<0.001 ; \mathrm{D} 21, p<0.001)$. Increased CD31 mRNA expression in the FE group $(p=0.033)$ and TE group ( $p=0.006$ ) was observed at D7 compared to the NC group, and the increased expression of mRNA continued until D21 (FE group, $p<0.001$; TE group, $p<0.001$ ) compared to the NC group. However, there was no significant difference between the experimental groups (Figure 5A). CD 31positive vessels were observed in the submucosa at D7 (Figure 5B).
VEGFR-3 mRNA expression was increased in all experimental groups at D3 compared to the NC group (FE group, $p=0.015$; TE group, $p=0.004$; TI group $p=0.001$ ) without significant differences between the experimental groups, and the increased expression of VEGFR-3 mRNA in the experimental groups was normalized at D7 (Figure 5C). Based on IHC staining, VEGFR-3-positive lymphatic vessels were observed in all experimental groups and these were limited at D3 (Figure 5D).

MMP-9 expression. The expression of MMP-9 mRNA was only increased at D21 in all experimental groups with significant differences compared to the NC group $(p<0.001$; TE group, $p<0.001$; TI group $p<0.001)$. In addition, the mRNA expression in the TE $(p=0.033)$ and TI $(p<0.001)$ groups was significantly higher than in the FE group (Figure 6A). The IF for MMP-9 showed the dominant expression of MMP-9 in the basal cell layer at D21 (Figure 6B). The MFI values of TE group $(p<0.001)$ and TI group $(p<0.001)$ were significantly higher than in NC and FE groups (Figure 6C). 
A. TGF- $\beta 1$ mRNA expressions

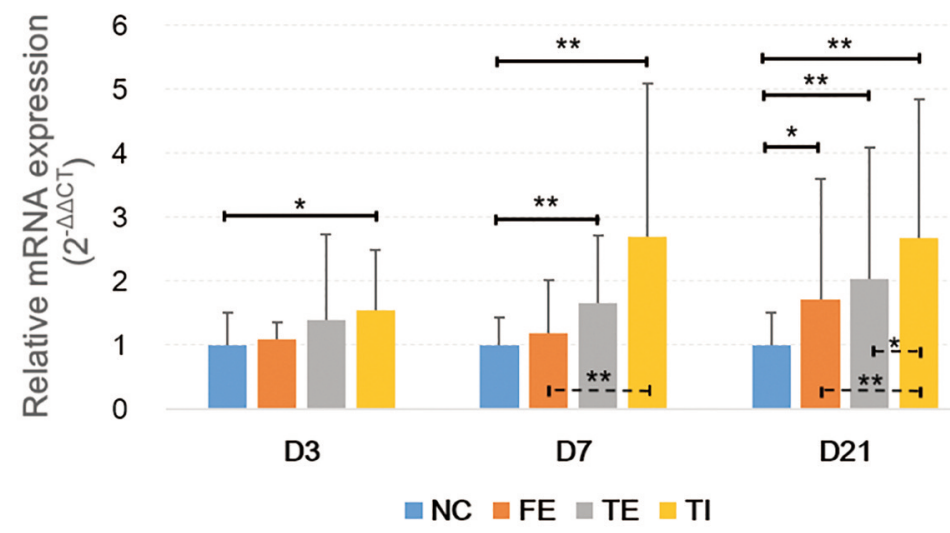

\section{B. Immunofluorescence for TGF- $\beta 1$}
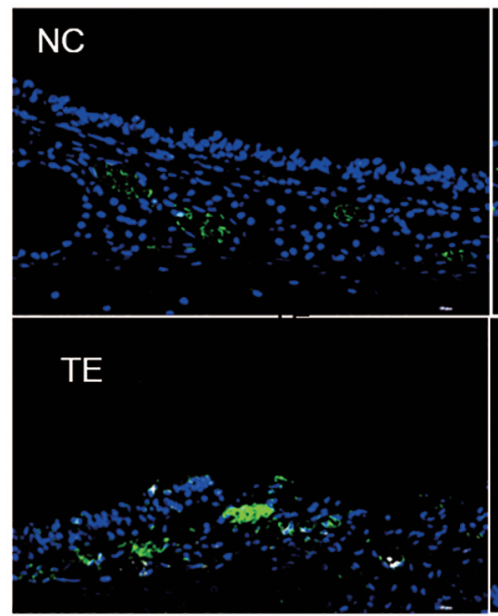

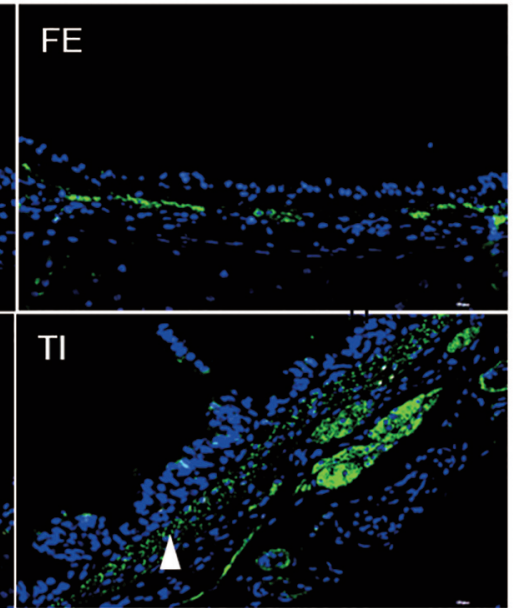

C. Immunofluorescence for TGF- $\beta 1$

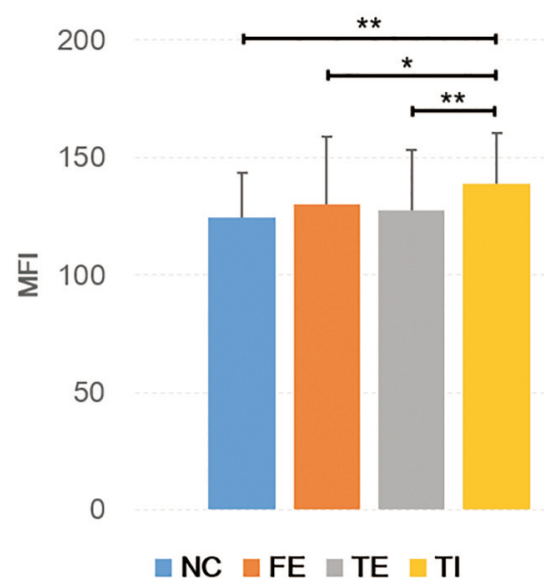

Figure 3. Transforming growth factor- $\beta 1$ (TGF- $\beta 1) m R N A$ and protein expression in the tracheal mucosa. (A) TGF- $\beta 1$ mRNA expression initially increased in the early phase (D3) after thyroid isthmusectomy (TI). Increased TGF- $\beta 1$ mRNA expression was observed in all surgical groups compared to the NC group in the late phase (D21). Significant differences in mRNA expression between the experimental groups were also observed in the late phase (D21). (B) TGF- $\beta 1$ protein was expressed in the submucosa and the basal epithelial layer (white arrowhead) in the late phase (D21) ('200 magnification). (C) The mean fluorescence intensity (MFI) of TGF- $\beta 1$ in the late phase. * $p<0.05$, ** $p<0.001$.

\section{Discussion}

We reported prolonged mucosal edema and functional changes in the TM after thyroid surgery even without direct mucosal injury induced by endotracheal intubation in a previous study (5). The results of this study revealed that thyroid surgery actually led to increased mRNA and protein expression of key regulators involved in the restoration of homeostasis for a considerable period after thyroid surgery.

The interpretation of TGF- $\beta 1$ expression requires attention because it plays a variety of roles depending upon its detection timing during the process of inflammation and homeostatic restoration $(8,11)$. TGF- $\beta 1$ primarily induces inflammation via leukocyte migration into the injured tissue in the early phase of homeostatic restoration and promotes
ECM protein expression and angiogenesis during the intermediate phase of homeostatic restoration (11-13). TGF$\beta 1$ interferes with the homeostatic restoration in the mucosa in the late phase by promoting collagen and extracellular protein deposition, and fibroblast proliferation, resulting in subepithelial fibrosis (14). In this study, increased TGF- $\beta 1$ mRNA expression was initially observed in the TI group, which underwent the most extensive surgery. A significant increase in mRNA expression was identified in all surgical groups at D21 compared to that of the $\mathrm{NC}$ group, and the degree of TGF- $\beta 1$ mRNA expression tended to increase with the extent of surgery. In particular, the MFI of TGF- $\beta 1$ was also increased according to the extent of surgery. These results imply that even if thyroid surgery was performed without endotracheal intubation, it interferes with the 


\section{A. HIF1- $\alpha$ mRNA expressions}

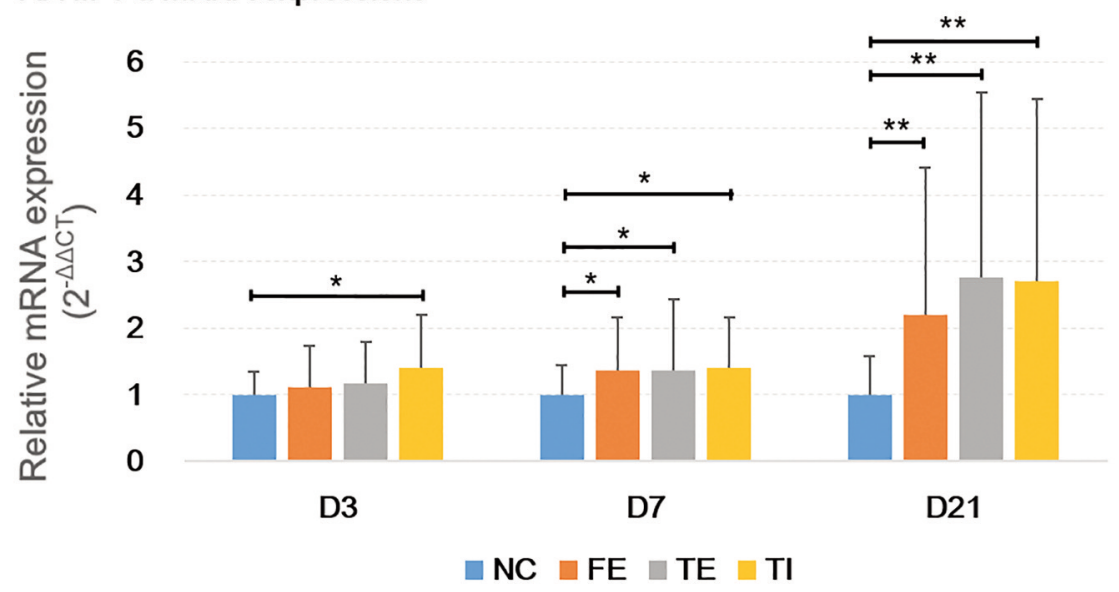

\section{B. Immunofluorescence for HIF1-a}
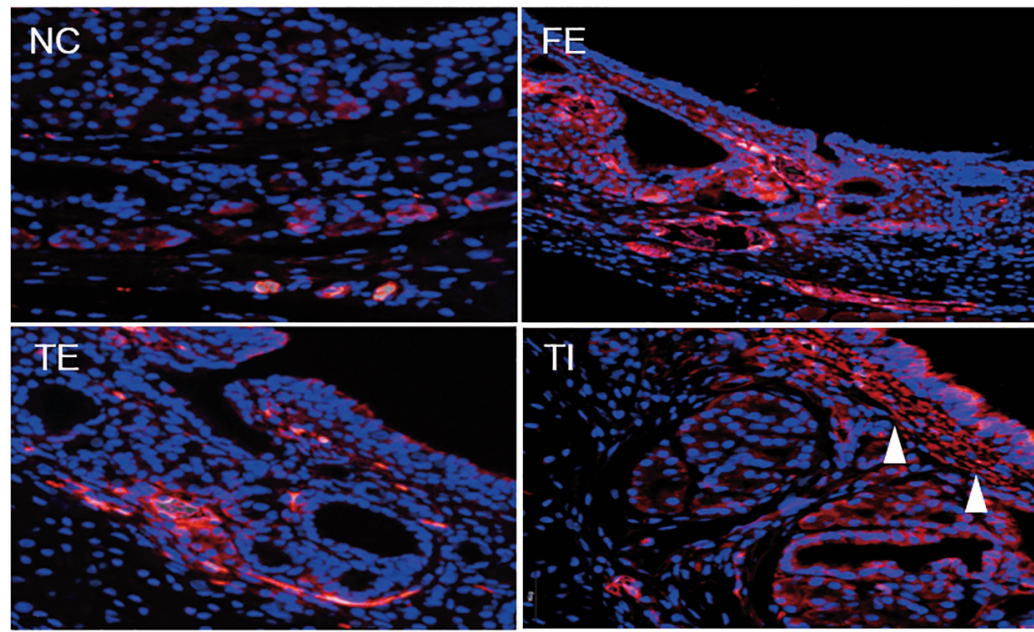

\section{Immunofluorescence for HIF1- $\alpha$}

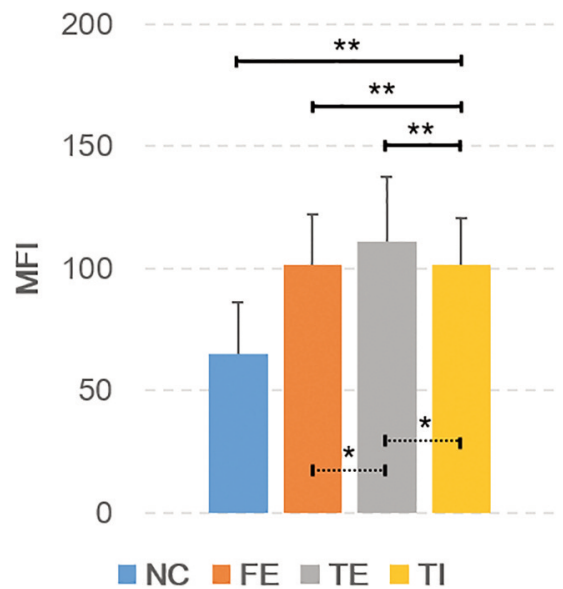

Figure 4. Specific hypoxia-inducible factor-1 $\alpha$ (HIF-1 $\alpha)$ mRNA and protein expression in the tracheal mucosa is shown. (A) Higher expression of HIF-1 $\alpha$ mRNA in the TI group than the NC group was initially observed in the early phase. The increased mRNA expression in all experimental groups was seen from the intermediate phase (D7) to the late phase (D21) without significant differences between the experimental groups. (B) $H I F-1 \alpha$ protein was predominantly expressed in the epithelium and submucosa (white arrowhead) from the intermediate phase (D7) after thyroid isthmusectomy. (C) Mean fluorescence intensity (MFI) of HIF-1 $\alpha$ in the intermediate phase. ${ }^{*} p<0.05$, ** $p<0.001$.

homeostasis of TM. In particular, the large extent of thyroid surgery is thought to have a negative effect on the homeostatic restoration of TM, which can lead to excessive ECM deposition in the submucosa.

Hypoxia is defined as the lack of necessary oxygen to meet the metabolic requirements of the cells and is a physiological process associated with stress such as inflammation or tissue injury (15). HIF- $1 \alpha$ is a transcription factor dynamically activated under low oxygen levels and TGF- $\beta 1$ expression in the injured tissue. In this study, TM hypoxia was predominantly observed in experimental groups than in NC group from D7, and HIF$1 \alpha$ protein was significantly expressed in epithelium and basal cell layers with significant differences in MFI between groups. Since HIF- $1 \alpha$ also contributes to TGF- $\beta 1$ expression in the late phase of homeostatic restoration, TM hypoxia primarily entails prolonged TGF- $\beta 1$ expression in TM and appears to be a significant factor inhibiting the restoration of TM homeostasis. However, the impact of the extent of thyroid surgery on TM hypoxia was not confirmed in this study.

Tissue hypoxia strongly leads to angiogenesis and increases oxygen and nutrients levels in the tissue $(16,17)$. Therefore, CD31 mRNA, a surface marker of endothelial cells in newly formed vessels, is also simultaneously increased with the expression of HIF-1 $\alpha$. CD 31-positive vessels were observed 
A. CD31 mRNA expressions

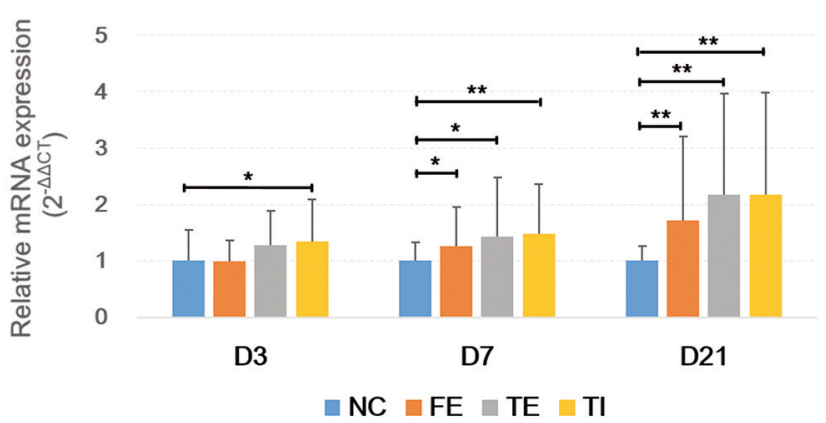

C. VEGFR-3 mRNA expressions

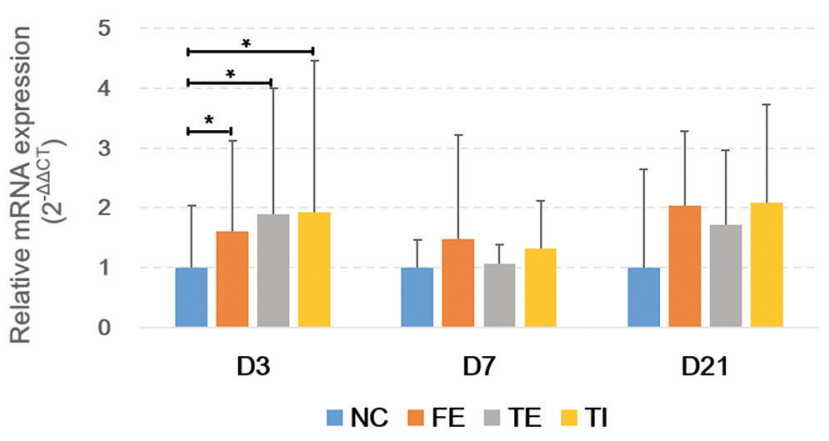

B. CD31 IHC staining

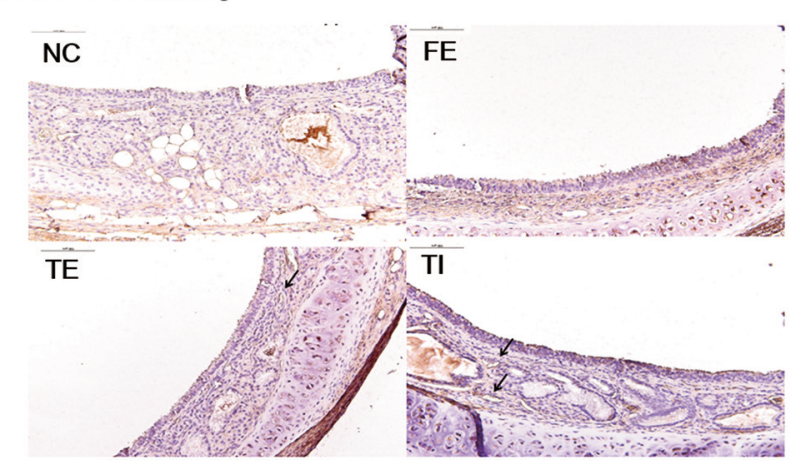

D. VEGFR-3 IHC staining

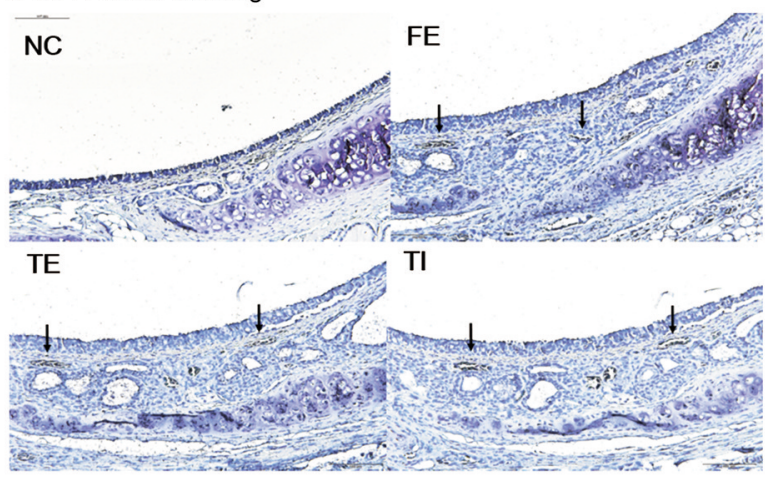

Figure 5. Expression of platelet endothelial cell adhesion molecule-1 (PECAM-1 also known as CD 31) and vascular endothelial growth factor receptor-3 (VEGFR-3) mRNA and protein in the tracheal mucosa is shown. (A) A higher CD31 mRNA expression in all experimental groups compared to the NC group was seen from the intermediate phase (D7) to the late phase (D21). (B) CD31-positive blood vessels (black arrows) were observed in the submucosa at the intermediate phase (D7). (C) The increased expression of VEGFR-3 mRNA in all experimental groups was limited to the early phase without significant difference between the experimental groups (D3). (D) VEGFR-3-positive lymphatic vessels (black arrows) were seen in the submucosa. $* p<0.05, * * p<0.001$.

in the submucosa from D7, probably in response to tissue hypoxia. Interestingly, lymphangiogenesis is activated via a pathway similar to that of angiogenesis in inflamed and injured tissues (18). However, increased VEGFR-3 mRNA expression and VEGFR-3-positive lymphatic vessels were only observed at D3, even though angiogenesis continued until D21. This phenomenon may be attributed to TGF- $\beta 1$ interfering with lymphatic endothelial cell proliferation, resulting in chronic intestinal fluid stasis after thyroid surgery (19).

MMP-9 is an enzyme that cleaves collagen, especially type IV collagen, the major component of airway basement membrane. This enzyme also enhances angiogenesis to restore tissue homeostasis (20). However, excessive expression of this enzyme is a marker of mucosal remodeling during the late phase of wound healing (21). In this study, MMP-9 protein expression in the basement membrane following increased MMP-9 mRNA expression was observed in the late phase after surgery. These results suggest excessive collagen deposition in the basement membrane, and differences in the degree of mRNA and protein expressions suggest that increased surgical extent may elevate the MMP-9 expression. It also continuous changes in TM up to 21 days after surgery.

In this study, even though thyroid surgery was performed without endotracheal intubation, actual protein expression following mRNA expression of key regulators of homeostatic restoration was found in the TM. The results of this study suggest that the morphological and functional changes of TM are a real phenomenon. In addition, more extensive dissection during thyroid surgery may interfere with homeostatic restoration of the TM following thyroid surgery for a considerable period of time after thyroid surgery. However, the results of this study must be interpreted with caution because of its limitations. First, the cause of impairment of homeostasis impairment in the TM after thyroid surgery is unclear whether it is caused by the thyroid surgery itself or by anesthesia for surgery. Second, the mismatch between mRNA and protein expression according to the time course after surgery and experimental groups, are presumed to occur due to the differences in 
A. MMP-9 mRNA expressions

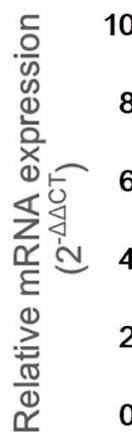

10

8

6

4

2

0

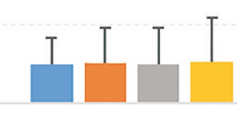

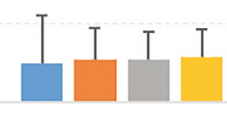

D7

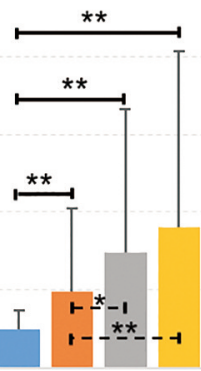

D21

$\square \mathrm{NC} \square \mathrm{FE} \square \mathrm{TE} \square \mathrm{TI}$

\section{B. Immunofluorescence for MMP-9}
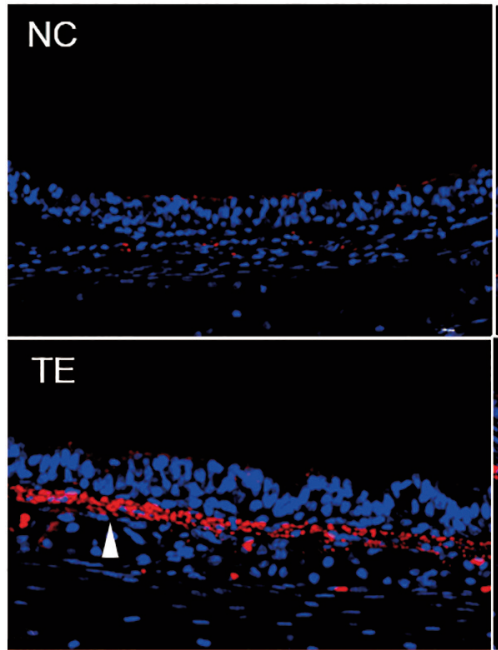

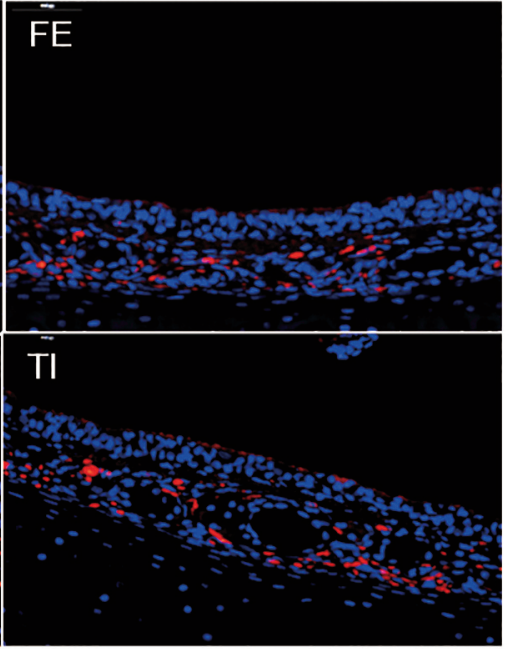

C. Immunofluorescence for MMP-9

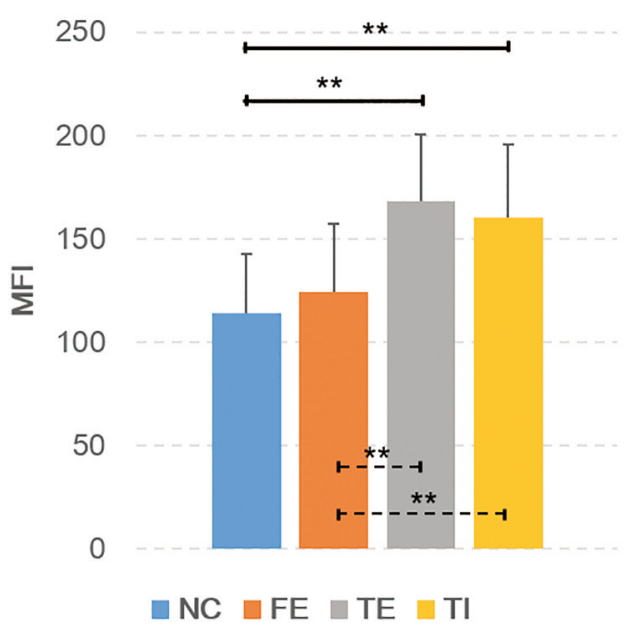

Figure 6. Matrix metalloproteinase-9 (MMP-9) mRNA expression and protein expression. (A) MMP-9 mRNA showed a higher expression in the experimental groups compared to the NC group, and a significant difference was detected between the experimental groups in the late phase (D21). (B) MMP-9 protein was expressed in the basal layer of the epithelium in the TE group (white arrowhead). The mean fluorescence intensity (MFI) of MMP-9 in the late phase. ${ }^{*} p<0.05, * * p<0.001$.

measurement of the areas of mRNA and protein expression. Third, although the mRNA expression of TGF- $\beta 1$ and MMP-9 varied substantially between the groups according to the extent of thyroid surgery, mRNA expression of other markers of TM hypoxia and angiogenesis did not show any significant difference between the experimental groups. Therefore, this study provides only limited information on how microcirculatory changes in the TM affect these chronic changes. Finally, since the increased mRNA and protein expression of key modulators continues until the late phase after thyroid surgery, further long-term studies are needed to establish airway remodeling caused by thyroid surgery, which is suggested as a cause of postthyroidectomy syndrome.

\section{Conclusion}

The thyroid surgery leads to an actual increase of TGF- $\beta 1$, HIF-1 $\alpha$, and MMP-9 expression in the TM, and more extensive thyroid surgery can further interfere with the homeostatic restoration of the TM for a considerable period of time after surgery. The results of this study suggest that the appropriate extent of thyroid surgery, not performing excessive dissection, may help restore the homeostasis of TM after thyroid surgery.

\section{Conflicts of Interest}

The Authors declare no conflicts of interest associated with this study. 


\section{Authors' Contributions}

Conception or design of the study: S.W.P and Y.S.L; data collection: H.B.K; data analysis and interpretation: B.H.K, J.H.P, and C.H.C; drafting the article: B.H K; critical revision of the article: B.H.K and Y.S.L.

\section{Acknowledgements}

This work was supported by the National Research Foundation of Korea (NRF) (grant number NRF-2017R1D1A1B03029710). Y.S.L and S.W.P contributed equally to this report as corresponding authors.

\section{References}

1 Whitsett JA: Airway epithelial differentiation and mucociliary clearance. Ann Am Thorac Soc 15(Suppl 3): S143-S148, 2018. PMID: 30431340. DOI: 10.1513/AnnalsATS.201802-128AW

2 Gohy ST, Hupin C, Pilette C and Ladjemi MZ: Chronic inflammatory airway diseases: the central role of the epithelium revisited. Clin Exp Allergy 46(4): 529-542, 2016. PMID: 27021118. DOI: $10.1111 /$ cea.12712

3 Branchett WJ and Lloyd CM: Regulatory cytokine function in the respiratory tract. Mucosal Immunol 12(3): 589-600, 2019. PMID: 30874596. DOI: 10.1038/s41385-019-0158-0

4 Bergeron C, Tulic MK and Hamid Q: Airway remodelling in asthma: from benchside to clinical practice. Can Respir J 17(4): e85-e93, 2010. PMID: 20808979. DOI: 10.1155/2010/318029

5 Lim YS, Choi YJ, Kim BH, Kim HB, Cho CG, Park SW and Park JH: Changes in tracheal respiratory mucosa after thyroidectomy: a rat model. In Vivo 34(3): 1133-1140, 2020. PMID: 32354902. DOI: 10.21873/invivo.11885

6 Furlow PW and Mathisen DJ: Surgical anatomy of the trachea. Ann Cardiothorac Surg 7(2): 255-260, 2018. PMID: 29707503. DOI: $10.21037 / \mathrm{acs} .2018 .03 .01$

7 Mieczkowski B and Seavey BF: Anatomy, Head and Neck, Trachea. In: StatPearls [Internet]. Treasure Island (FL): StatPearls Publishing. Available at: https://www.ncbi.nlm.nih.gov/books/ NBK448070 [Last accessed on September 26, 2021]

8 Halwani R, Al-Muhsen S, Al-Jahdali H and Hamid Q: Role of transforming growth factor- $\beta$ in airway remodeling in asthma. Am J Respir Cell Mol Biol 44(2): 127-133, 2011. PMID: 20525803. DOI: $10.1165 / \mathrm{rcmb} .2010-0027 \mathrm{TR}$

9 Imtiyaz HZ and Simon MC: Hypoxia-inducible factors as essential regulators of inflammation. Curr Top Microbiol Immunol 345: 105-120, 2010. PMID: 20517715. DOI: 10.1007/ 82_2010_74

10 Atkinson JJ and Senior RM: Matrix metalloproteinase-9 in lung remodeling. Am J Respir Cell Mol Biol 28(1): 12-24, 2003. PMID: 12495928. DOI: $10.1165 / \mathrm{rcmb} .2002-0166$ TR

11 Pakyari M, Farrokhi A, Maharlooei MK and Ghahary A: Critical role of transforming growth factor beta in different phases of wound healing. Adv Wound Care (New Rochelle) 2(5): 215-224, 2013. PMID: 24527344. DOI: 10.1089/wound.2012.0406
12 Ferrari G, Cook BD, Terushkin V, Pintucci G and Mignatti P: Transforming growth factor-beta 1 (TGF-beta1) induces angiogenesis through vascular endothelial growth factor (VEGF)-mediated apoptosis. J Cell Physiol 219(2): 449-458, 2009. PMID: 19180561. DOI: 10.1002/jcp.21706

13 Boxall C, Holgate ST and Davies DE: The contribution of transforming growth factor-beta and epidermal growth factor signalling to airway remodelling in chronic asthma. Eur Respir J 27(1): 208-229, 2006. PMID: 16387953. DOI: 10.1183/ 09031936.06 .00130004

14 Kenyon NJ, Ward RW, McGrew G and Last JA: TGF-beta1 causes airway fibrosis and increased collagen I and III mRNA in mice. Thorax 58(9): 772-777, 2003. PMID: 12947136. DOI: 10.1136/thorax.58.9.772

15 Eltzschig HK and Carmeliet P: Hypoxia and inflammation. N Engl J Med 364(7): 656-665, 2011. PMID: 21323543. DOI: 10.1056/NEJMra0910283

16 Mingyuan X, Qianqian P, Shengquan X, Chenyi Y, Rui L, Yichen $S$ and Jinghong X: Hypoxia-inducible factor- $1 \alpha$ activates transforming growth factor- $\beta 1 / \mathrm{Smad}$ signaling and increases collagen deposition in dermal fibroblasts. Oncotarget 9(3): 31883197, 2017. PMID: 29423039. DOI: 10.18632/oncotarget. 23225

17 Copple BL: Hypoxia stimulates hepatocyte epithelial to mesenchymal transition by hypoxia-inducible factor and transforming growth factor-beta-dependent mechanisms. Liver Int 30(5): 669-682, 2010. PMID: 20158611. DOI: 10.1111/ j.1478-3231.2010.02205.x

18 Liao $\mathrm{S}$ and von der Weid PY: Inflammation-induced lymphangiogenesis and lymphatic dysfunction. Angiogenesis 17(2): 325-334, 2014. PMID: 24449090. DOI: 10.1007/s10456014-9416-7

19 Clavin NW, Avraham T, Fernandez J, Daluvoy SV, Soares MA, Chaudhry A and Mehrara BJ: TGF-beta1 is a negative regulator of lymphatic regeneration during wound repair. Am J Physiol Heart Circ Physiol 295(5): H2113-H2127, 2008. PMID: 18849330. DOI: 10.1152/ajpheart.00879.2008

20 Rundhaug JE: Matrix metalloproteinases, angiogenesis, and cancer: commentary re: A. C. Lockhart et al., Reduction of wound angiogenesis in patients treated with BMS-275291, a broad spectrum matrix metalloproteinase inhibitor. Clin Cancer Res., 9: 00-00, 2003. Clin Cancer Res 9(2): 551-554, 2003. PMID: 12576417.

21 Fingleton B: Matrix metalloproteinases as regulators of inflammatory processes. Biochim Biophys Acta Mol Cell Res 1864(11 Pt A): 2036-2042, 2017. PMID: 28502592. DOI: 10.1016/j.bbamcr.2017.05.010 